- promotion: that future health care planning and end of life discussions are normalised in society

- resources: ACP is accessible to all regardless of language, literacy level or cultural beliefs

- education and training: we have workforce and community prepared to have conversations and use ACPs

- monitoring and evaluation: we know care is based on what matters to consumers

- implementation: we are maximising value for DHBs

Result a national mandate, strategy and specified actions have resulted in an increase in ACP activity; increased governance with decision-making being supported by a representative Steering Group; wider national stakeholder engagement and buy in from agencies in and outside of the health sector.

\section{OP80 SHARED CARE PLANNING: A NEW MODEL TO INTEGRATE ADVANCE CARE PLANNING INTO COMMUNITY. THE BASQUE COUNTRY EXPERIENCE}

I Saralegui*. Organización Sanitaria Integrada Araba, Vitoria-Gasteiz, Spain

\subsection{6/spcare-2019-ACPICONGRESSABS.80}

In the Basque Country, a project has been implemented based on community education, the training of health and social workers. It tries to elicit the individual's preferences and encourages them to take part in planning their care. Writing down advance directives is not the main aim.

The target population is chronic patients, elderly people and anyone at the end of life. The project also includes everyone who wants to think about the process of dying and needs to have a conversation related to this topic.

The project started in 2014 as a bottom-up project and it has grown into a top-down project performed in the whole Basque Health Service.

Results More than one hundred conferences and debates have been taking place in neighborhood associations, cultural centers, libraries or educational centers. More than five thousand people have attended these activities. One thousand workers have attended a basic training course, more than 500 workers have been trained as SCP facilitators and more than 700 doctors and nurses have participated in conversations with patients and families helped by an SCP facilitator. In $75 \%$ of cases, the citizens asked to be included after attending a conference; only $25 \%$ of participants were included because of a doctor or nurse's invitation. Many support documents have been created in order to explain the project and to make it easier to understand.

The keystone is the training of health and social workers in order to answer citizens' requests and to integrate the conversations into everyday care.

\section{OP81 IMPLEMENTATION OF ADVANCE CARE PLANNING IN AGED CARE: WHAT DO YOU WANT AND HOW CAN WE HELP?}

${ }^{1} \mathrm{H}$ Kelly, ${ }^{1} \mathrm{~L}$ Nolte, ${ }^{2} \mathrm{M}$ Fearn, ${ }^{2} \mathrm{~F}$ Batchelor, ${ }^{2} \mathrm{~B}$ Haralambous, ${ }^{2} \mathrm{P}$ Mackell, ${ }^{2} \mathrm{~K}$ Hwang, ${ }^{1} \mathrm{~K}$ Detering. ${ }^{1}$ Advance Care Planning Australia, Melbourne, Australia; ${ }^{2}$ National Ageing Research Institute Inc, Melbourne, Australia

10.1136/spcare-2019-ACPICONGRESSABS.81
Introduction The goal of advance care planning (acp) is to ensure that individuals receive future care consistent with their expressed preferences. Benefits of acp include: increased adherence to a person's preferences, higher staff satisfaction, reductions in unwanted hospitalisations and medical treatments, and reduced stress and anxiety for family. Despite clear benefits, uptake of acp has been limited in Australian aged care settings. This research explored barriers and enablers to implementing acp in residential and community aged care settings, from the perspectives of aged care staff and older people.

Method Focus groups and interviews with aged care staff and older people were conducted in four Australian states. Sixty staff from 15 residential and community aged care organisations and 24 older people participated. A thematic analysis was undertaken to identify facilitators and barriers.

Results Factors identified as impacting on the experiences and implementation of acp included: knowledge and understanding access to education/training understanding of relevant legislation having defined roles/responsibilities cognitive capacity of the older person timing of initiating the conversation availability of clear policies/procedures engaging older people and their families diversity within the workforce and older people.

Conclusions Aged care staff indicated there are a range of factors that can impact on acp implementation. All these factors may need to be considered when implementing acp within Australian aged care organisations. The findings informed the development of a new national resource titled Advance care planning in aged care: a guide to support implementation in community and residential settings.

\section{OP82 IDENTIFICATION, IMPLEMENTATION AND EVALUATION OF INDICATORS TO MONITOR SUCCESSFUL UPTAKE OF ADVANCE CARE PLANNING IN ALBERTA}

${ }^{1,2} \mathrm{~J}_{\mathrm{Xiao}}{ }^{*}{ }^{3} \mathrm{~J}$ SimOn, ${ }^{4} \mathrm{~T}$ Lynn Wityk Martin, ${ }^{4} \mathrm{~S}$ Iversen, ${ }^{1} \mathrm{M}$ Douglas, ${ }^{1} \mathrm{~A}$ Potapov, ${ }^{1} \mathrm{M}$ Nesari, ${ }^{3} \mathrm{P}$ Biondo, ${ }^{3} \mathrm{~A}$ Kanters, ${ }^{1,2} \mathrm{~K}$ Fassbender. ${ }^{1}$ University of Alberta, Edmonton, Canada; ${ }^{2}$ Covenant Health Palliative Institute, Edmonton, $A B$, Canada; ${ }^{3}$ University of Calgary, Calgary, Canada; ${ }^{4}$ Alberta Health Services, Edmonton, $A B$, Canada

\subsection{6/spcare-2019-ACPICONGRESSABS.82}

Background In 2014, a province-wide policy for advance care planning (ACP) and Goals of Care Designation (GCD) was implemented in Alberta, Canada; nevertheless, few quality indicators have been rigorously developed or evaluated for measuring the uptake of ACP/GCD.

Methods In phase I, we performed a systematic literature review and environmental scan to identify potential ACP/GCD indicators. A Delphi consensus-based approach, consisting of 3 rounds of face-to-face meetings and/or online surveys, was used to develop a short list of indicators. In phase II, the panelists met face-to-face to operationalize and implement the indicators. In phase III, two validated questionnaires and semistructured interviews of 60 individuals (stratified by manager/ practitioner and physician/nurse) are being used to evaluate the usability and acceptability of the implemented indicators on a dashboard interface.

Results A total of 132 potential indicators were identified in phase I. The indicators were reduced and refined to 18 after 3 Delphi rounds. Phase II resulted in 9 valid and feasible indicators in a measurable format (i.e. numerator, denominator, data source defined). The Phase III protocol is under 
ethical review and potential participants' recruitment is underway.

Conclusions of 132 quality indicators for ACP/GCD, 9 are feasible, valid, usable and acceptable for monitoring performance in the rollout of ACP/GCD. This set of indicators shows promise for describing and evaluating ACP/GCD uptake throughout a complex, multi-sector healthcare system.

\section{OP83 THE CHALLENGE OF FINDING THE 'RIGHT' OUTCOME TO MEASURE THE EFFECTS OF ACP - PART I}

1J Rietjens*, ${ }^{2} \mathrm{R}$ Sudore, ${ }^{1} \mathrm{~A}$ van der Heide, ${ }^{3} \mathrm{D}$ Heyland, ${ }^{1} \mathrm{I}$ Korfage. ${ }^{1}$ Erasmus University Medical Centre, Rotterdam, Netherlands; ${ }^{2}$ University of California, San Fransisco, USA; ${ }^{3}$ Queen's University, Kingston, Canada

10.1136/spcare-2019-ACPICONGRESSABS.83

Background Advance care planning (ACP) is increasingly implemented in oncology and beyond, but a definition of ACP and recommendations concerning its use are lacking. This hinders the development of ACP programmes and the evaluation of ACP's effectiveness.

Methods We used a formal Delphi consensus process to help develop a definition of ACP and provide recommendations for its application in healthcare, policy and research.

Results Of the 109 experts (82 from Europe, 16 from North America, and 11 from Australia) who rated the ACP definitions and its 41 recommendations, agreement for each definition or recommendation was between $68-100 \%$. ACP was defined as the ability to enable individuals to define goals and preferences for future medical treatment and care, to discuss these goals and preferences with family and health-care providers, and to record and review these preferences if appropriate. Recommendations included the adaptation of ACP based on the readiness of the individual; targeting ACP content as the individual's health condition worsens; and, using trained non-physician facilitators to support the ACP process. A list of outcome measures is also presented to enable the pooling and comparison of results of ACP studies.

Conclusion This large international Delphi panel was able to come to a consensus on an ACP definition and recommendations. This represents an important first step in providing clarity with a view to further policy and research in this field. We believe that our recommendations can provide guidance for clinical practice, ACP policy, and research.

\section{OP84 THE CHALLENGE OF FINDING THE 'RIGHT' OUTCOME TO MEASURE THE EFFECTS OF ACP - PART II}

${ }^{1} \mathrm{R}$ Sudore, ${ }^{2} \mathrm{D}$ Heyland, ${ }^{3} \mathrm{H}$ Lum, ${ }^{4} \mathrm{~J}$ Rietjens, ${ }^{4} \mathrm{~K}$ Korfage ${ }^{*},{ }^{5} \mathrm{M}$ Howard, ${ }^{5} \mathrm{~J}$ You. ${ }^{1}$ University of California, San Fransisco, USA; ${ }^{2}$ Queen's University, Kingston, Canada; ${ }^{3}$ Denver, Colorado, USA; ${ }^{4}$ Erasmus University Medical Center, Rotterdam, Netherlands; ${ }^{5}$ McMaster University, Hamilton, Ontario, Canada

\subsection{6/spcare-2019-ACPICONGRESSABS.84}

Background Standardized outcomes that define successful advance care planning (ACP) are lacking. The objective of this study was to create an Organizing Framework of ACP outcome constructs and rate the importance of these outcomes.
Methods This study convened a Delphi panel consisting of 52 multidisciplinary, international ACP experts including clinicians, researchers, and policy leaders from four countries. We conducted literature reviews and solicited attendee input from five international ACP conferences to identify initial ACP outcome constructs. In five Delphi rounds, we asked panelists to rate patient-centered outcomes on a seven-point "not-at-all" to "extremely important" scale. We calculated means and analyzed panelists' input to finalize an Organizing Framework and outcome rankings.

Results Organizing Framework outcome domains included process (e.g., attitudes), actions (e.g., discussions), quality of care (e.g., satisfaction), and health care (e.g., utilization). The top five outcomes included 1) care consistent with goals, mean 6.71 ( \pm SD 0.04); 2) surrogate designation, 6.55 (0.45); $3)$ surrogate documentation, $6.50(0.11)$; 4) discussions with surrogates, 6.40 (0.19); and 5) documents and recorded wishes are accessible when needed 6.27 (0.11). Advance directive documentation was ranked 10th, 6.01 (0.21). Panelists raised caution about whether "care consistent with goals" can be reliably measured.

Conclusion A large, multidisciplinary Delphi panel developed an Organizing Framework and rated the importance of ACP outcome constructs. Top rated outcomes should be used to evaluate the success of ACP initiatives. More research is needed to create reliable and valid measurement tools for the highest rated outcomes, particularly "care consistent with goals".

\section{OP85 THE CHALLENGE OF FINDING THE 'RIGHT' OUTCOME TO MEASURE THE EFFECTS OF ACP: PART III}

S Hickman* , K Unroe, A Torke. Indiana University, Indianapolis, USA

\subsection{6/spcare-2019-ACPICONGRESSABS.85}

Background A key outcome of successful advance care planning is consistency between patient goals of care and the care provided. However, assessing consistency is methodologically challenging due to conceptual and logistical barriers as well as practice limitations.

Methods The team reviewed the literature and identified key methodological barriers to measuring care consistency with patient preferences and goals of care. Strategies to overcome these barriers are proposed.

Results Key methodological barriers include: 1) the specificity and relevance of documentation about preferences and goals of care; 2) the availability of documentation about preferences and goals of care; 3 ) the stability of documented preferences and goals of care; 4) the timing of treatments provided and identifying decisions not to treat ("nonevents"); and 5) calculating rates of consistency. Strategies to address these methodological barriers include using tools to document specific preferences, incorporation into electronic health records, period reassessment of preferences, prospective data collection, and using percent agreement to report consistency.

Discussion There are clear challenges to assessing care consistency with preferences and goals of care. The ability to assess and report on this important outcome of successful advance care planning requires focused efforts to improve practice. 